\title{
Thermodynamics and Intermolecular Interactions during the Insertion of Anionic Naproxen into Model Cell Membranes
}

\author{
Natalia Rojas-Valencia, ${ }^{\S},, \ddagger$ Sara Gómez, ${ }^{\Uparrow}$ Francisco Núñez-Zarur, ${ }^{\dagger}$ \\ Chiara Cappelli, "Cacier Hadad, ${ }^{\S}$ Albeiro Restrepo ${ }^{\S}$ \\ §Instituto de Química, Universidad de Antioquia UdeA, Calle 70 No. 52-21, Medellín, \\ Colombia \\ ${ }^{\dagger}$ Facultad de Ciencias Básicas, Universidad de Medellín, Carrera 87 No. 30-65, Medellín, \\ Colombia \\ ${ }^{\ddagger}$ Escuela de Ciencias y Humanidades, Departamento de Ciencias Básicas, Universidad Eafit, \\ AA 3300, Medellín, Colombia \\ `Scuola Normale Superiore, Classe di Scienze, Piazza dei Cavalieri 7, 56126, Pisa, Italy \\ ${ }^{*}$ Correspondence: nandrea.rojas@udea.edu.co, albeiro.restrepo@udea.edu.co
}

\section{Supplementary information}

\section{Contents}

1 MD simulations $\quad$ S2

2 Charge redistribution $\quad$ S3

$\begin{array}{lll}3 & \text { QTAIM descriptors } & \text { S4 }\end{array}$ 


\section{MD simulations}

Table S1: Conditions for the MD simulations of the anionic naproxen into the lipid bilayer environment

\begin{tabular}{|c|c|}
\hline Item & Description \\
\hline MD package & Gromacs 5.02[1] \\
\hline Naproxen Force field & CGenFF $[2,3]$ \\
\hline Lipid Force field & CHARMM36[4] \\
\hline Water molecules & TIP3P $[5]$ \\
\hline Energy minimization & steepest descent minimization algorithm \\
\hline NVT equilibration & $\begin{array}{l}\text { total time: } 0.05 \mathrm{~ns} \\
\text { velocity-rescaling method }[6] \\
\text { integration time step: } 0.2 \mathrm{fs}\end{array}$ \\
\hline NPT equilibration & $\begin{array}{l}\text { total time: } 0.175 \mathrm{~ns} \\
\text { integration time step: } 1 \text { fs } \\
\text { barostat: Berendsen[7] }\end{array}$ \\
\hline $\begin{array}{c}\text { Initial configurations } \\
\text { (Steered molecular dynamics (SMD)) }\end{array}$ & $\begin{array}{l}\text { integration time step: } 2 \mathrm{fs} \\
\text { LINCS algorithm[8] } \\
\text { electrostatic interactions: Particle Mesh Ewald (PME)[9] } \\
\text { pulling rate: } 0.008 \mathrm{~nm} \mathrm{ps}{ }^{-1} \\
\text { harmonic constant: } \mathrm{k}=1000 \mathrm{~kJ} \mathrm{~mol}^{-1} \mathrm{~nm}^{-2} \\
\text { Lennard-Jones cut-off: } 1.2 \mathrm{~nm} \\
\text { Coulomb cut-off: } 1.2 \mathrm{~nm} \\
\text { leap-frog algorithm } \\
\text { thermostat: Nosé-Hoover[10] } \\
\text { barostat: Parrinello-Rahman[1]] }\end{array}$ \\
\hline $\begin{array}{c}\text { NPT production } \\
\text { (Umbrella sampling method }[12])\end{array}$ & $\begin{array}{l}\text { total time: } 840 \mathrm{~ns} \\
\text { integration time step: } 2 \mathrm{fs} \\
\text { LINCS algorithm[8] } \\
\text { harmonic constant: } \mathrm{k}=1000 \mathrm{~kJ} \mathrm{~mol}^{-1} \mathrm{~nm}^{-2} \\
\text { electrostatic interactions: Particle Mesh Ewald (PME)[9] } \\
\text { Lennard-Jones cut-off: } 1.2 \mathrm{~nm} \\
\text { Coulomb cut-off: } 1.2 \mathrm{~nm} \\
\text { leap-frog algorithm } \\
\text { thermostat: Nosé-Hoover[10] } \\
\text { barostat: Parrinello-Rahman[1]] }\end{array}$ \\
\hline
\end{tabular}




\section{Charge redistribution}
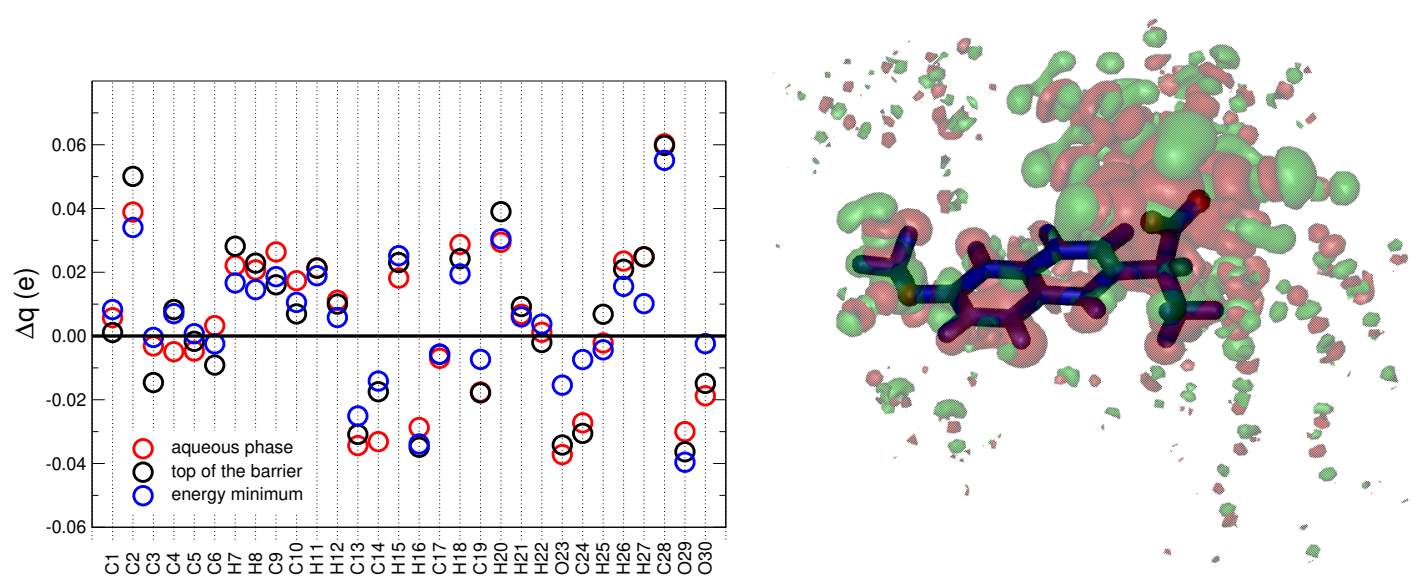

Figure S1: Left: average natural relative charges on anionic Naproxen atoms (relative to isolated $\mathrm{NAP}^{-}$, solid line at $\Delta q=0$ ) for the three equilibrium positions in the free energy profile (Figure 3) for the insertion of anionic Naproxen into model DMPC membranes. All charges derived from CAM-B3LYP/6-31+G(d) computations on all available snapshots. See Figure 1 for atom numbers. Right: Difference in the electron density from the tertiary $\mathrm{NAP}^{-} /$membrane/water system and the isolated components taking one particular snapshot at the energy minimum. Red surfaces indicate regions of loss of electron density, green surfaces indicate regions of gain of electron density. 


\section{QTAIM descriptors}
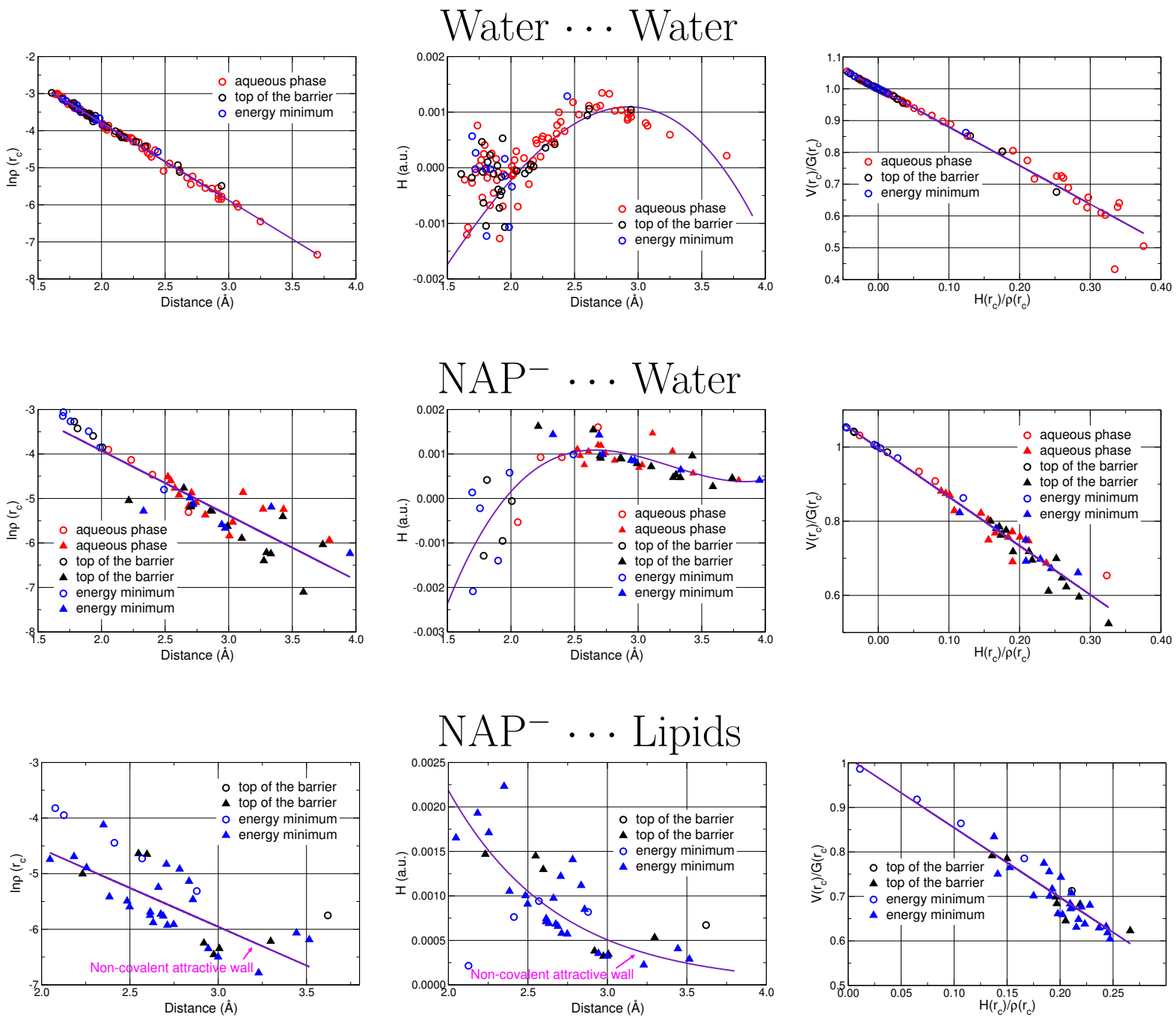

Figure S2: Properties of the bond critical points for intermolecular interactions in randomly selected frames (same frames as in the NCI (Figure 4), and NBO (Figure 5) analyses.) Top: Water to water interactions. Middle: $\mathrm{Nap}^{-}$to water interactions. Bottom: $\mathrm{Nap}^{-}$to lipid interactions. In all cases, open symbols refer to interactions between the polar region of $\mathrm{Nap}^{-}$and the environment while filled symbols refer to interactions between the non-polar region of $\mathrm{Nap}^{-}$and the environment.

\section{References}

[1] M. J. Abraham, T. Murtola, R. Schulz, S. Páll, J. C. Smith, B. Hess, and E. Lindahl, "Gromacs: High performance molecular simulations through multi-level parallelism from laptops to supercomputers," SoftwareX, vol. 1, pp. 19-25, 2015. 
[2] K. Vanommeslaeghe and A. D. MacKerell, "Automation of the charmm general force field (cgenff) i: Bond perception and atom typing," J. Chem. Inf. Model, vol. 52, no. 12, pp. 3144-3154, 2012. PMID: 23146088.

[3] K. Vanommeslaeghe, E. P. Raman, and A. D. MacKerell, "Automation of the charmm general force field (cgenff) ii: Assignment of bonded parameters and partial atomic charges," J. Chem. Inf. Model, vol. 52, no. 12, pp. 3155-3168, 2012. PMID: 23145473.

[4] J. B. Klauda, R. M. Venable, J. A. Freites, J. W. O'Connor, D. J. Tobias, C. MondragonRamirez, I. Vorobyov, A. D. MacKerell, and R. W. Pastor, "Update of the charmm all-atom additive force field for lipids: Validation on six lipid types," J. Phys. Chem. $B$, vol. 114, no. 23, pp. 7830-7843, 2010. PMID: 20496934.

[5] W. L. Jorgensen, J. Chandrasekhar, J. D. Madura, R. W. Impey, and M. L. Klein, "Comparison of simple potential functions for simulating liquid water," J. Chem. Phys., vol. 79, no. 2, pp. 926-935, 1983.

[6] G. Bussi, D. Donadio, and M. Parrinello, "Canonical sampling through velocity rescaling," J. Chem. Phys., vol. 126, no. 1, p. 014101, 2007.

[7] H. J. C. Berendsen, J. P. M. Postma, W. F. van Gunsteren, A. DiNola, and J. R. Haak, "Molecular dynamics with coupling to an external bath," J. Chem. Phys., vol. 81, no. 8, pp. 3684-3690, 1984.

[8] B. Hess, H. Bekker, H. J. C. Berendsen, and J. G. E. M. Fraaije, "Lincs: A linear constraint solver for molecular simulations," J. Comput. Chem., vol. 18, no. 12, pp. 1463$1472,1997$.

[9] U. Essmann, L. Perera, M. Berkowitz, T. Darden, H.-C. Lee, and L. Pedersen, "A smooth particle mesh ewald method," Int. J. Chem. Phys., vol. 103, pp. 8577-8593, 1995.

[10] S. Nosé, "A molecular dynamics method for simulations in the canonical ensemble," Mol. Phys., vol. 52, no. 2, pp. 255-268, 1984.

[11] M. Parrinello and A. Rahman, "Polymorphic transitions in single crystals: A new molecular dynamics method," Int. J. Appl. Phys., vol. 52, no. 12, pp. 7182-7190, 1981.

[12] J. Kästner, "Umbrella sampling," Wiley Interdiscip. Rev. Comput. Mol. Sci., vol. 1, no. 6, pp. 932-942, 2011. 